\title{
On the Approximation of the Generalized- $K$ PDF by a Gamma PDF Using the Moment Matching Method
}

\author{
Saad Al-Ahmadi \\ Systems \& Computer Engineering \\ Carleton University \\ Ottawa, Canada \\ saahmadi@sce.carleton.ca
}

\author{
Halim Yanikomeroglu \\ Systems \& Computer Engineering \\ Carleton University \\ Ottawa, Canada \\ halim@sce.carleton.ca
}

\begin{abstract}
Using the Nakagami probability density function (PDF) to model multipath fading and the Gamma PDF to model shadowing, in a wireless channel, has led to a closed-form expression for the composite fading PDF, known as the generalized-K PDF (also called Gamma-Gamma PDF). However, further derivations have shown that the cumulative distribution function (CDF) and the characteristic function of the generalized$K$ PDF contain special functions that are involved to handle. In this paper, an approximation of the generalized- $K$ PDF by the familiar Gamma PDF is introduced. The parameters of the approximating Gamma PDF are computed using the moment matching method. The accuracy of this approximation in the lower and upper tail regions is enhanced by adjusting the parameters of the approximating Gamma distribution in each region. The CDF and the complementary CDF plots show that this approximation is sufficiently accurate for both integer and non-integer practical values of the multipath fading and shadowing parameters. The region-wise approximation obtained by the adjusted moment matching method is used to wellapproximate the PDF of the sum of identically and independent generalized- $K$ random variables. Applications of the obtained results arise in distributed antenna systems (DASs), cooperative relay networks, radar, and sonar systems.
\end{abstract}

Keywords; Composite fading, generalized- $K$ distribution; Gamma distribution; moment matching; lower and upper tails; distributed antenna systems; relay networks.

\section{INTRODUCTION}

In wireless channels, the phenomena of multipath fading and shadowing take place simultaneously leading to composite fading. The small-scale multipath fading is usually modeled using Rayleigh, Rician, or Nakagami distributions. The latter one is general enough to encompass the Rayleigh distribution as a special case and to well-approximate the Rician distribution. Large-scale (shadow) fading is usually modeled using the lognormal distribution [1]. However, the lognormalbased composite fading models do not lead to closed form expressions of the received signal power probability density function (PDF) which hampers further analytical derivations. As an alternative, it has been proposed to use the Gamma distribution to model the average power random variations due to shadowing $[2,3]$ and it was shown using experimental data that it models shadow fading as close as the lognormal distribution [3]. Assuming that the multipath fading and shadowing are independent and using the fact that the square of a Nakagami random variable (RV) is Gamma distributed, a closed-form expression for the composite fading PDF was developed in the form of a Gamma-Gamma (generalized- $K$ ) PDF. However, further derivations using that PDF have shown to be computationally involved or analytically difficult due to the involvement of special functions. In this paper, an adjusted form of the first-two moment matching method is introduced to approximate the generalized- $K$ composite fading PDF by a simple Gamma PDF. The introduced approximation can be tight in the upper and lower tail regions for various integer and non-integer values of the multipath fading and shadowing parameters. The introduced approximation provides a simplifying model for the composite fading in wireless communication systems, scattering in radar and, reverberation in sonar.

\section{COMPOSITE FADING MODEL}

The envelope of the received signal, due to multipath fading, can be modeled by the versatile Nakagami distribution. Subsequently, the PDF of the received power $\gamma$, conditioned on the average power $\Omega$, is a Gamma PDF of the form:

$p_{\gamma / \Omega}(x)=\frac{1}{\Gamma\left(m_{m}\right)}\left(\frac{m_{m}}{\Omega}\right)^{m} x^{m-1} \exp \left\{-\frac{m_{m} x}{\Omega}\right\}, x \geq 0, m_{m} \geq 0.5$,

where $\Gamma(\cdot)$ is the Gamma function and $m_{m}$ is the Nakagami multipath fading parameter. The variation of the average power, due to shadowing, is usually modeled by the lognormal distribution, however, in this paper, the Gamma distribution, which has shown a good fit to experimental data $[3,4]$ and can approximate the lognormal distribution, is used [5-7]:

$$
p_{\Omega}(y)=\frac{1}{\Gamma\left(m_{s}\right)}\left(\frac{m_{s}}{\Omega_{0}}\right)^{m_{s}} y^{m_{s}-1} \exp \left\{-\frac{m_{s} y}{\Omega_{0}}\right\}, y \geq 0, m_{s}>0 \text {. }
$$

In (2), the parameter $m_{s}$ is the shadowing parameter and $\Omega_{0}$ is a measure of the average power [6]. The PDFs ranging from 
lognormal to Gaussian can be generated by varying the shadowing parameter $m_{s}$ [5]. Similar to the multipath fading parameter $m_{m}$, the severity of shadowing is inversely proportional to $m_{\mathrm{s}}$ so that small values of $m_{s}$ indicate severe shadowing conditions. In $[3,6]$, using the moment matching method between the PDF in (2) and the lognormal PDF, it was shown that $m_{s}=\frac{1}{e^{\left(\sigma_{s} / 8.686\right)^{2}}-1}$ where $\sigma_{\mathrm{s}}$ denotes the standard deviation of the corresponding lognormal PDF.

The PDF of the received signal power due to both multipath fading and shadowing, can be obtained as [1]

$$
p_{\gamma}(x)=\int_{0}^{\infty} p_{\gamma / \Omega}(x / y) p_{\Omega}(y) d y .
$$

Inserting (1) and (2) in (3) yields [5, 7]

$$
\begin{aligned}
p_{\gamma}(x) & =\frac{2}{\Gamma\left(m_{s}\right) \Gamma\left(m_{m}\right)}\left(\frac{b}{2}\right)^{m_{s}+m_{m}} x^{\left(\frac{m_{s}+m_{m}}{2}\right)-1} K_{m_{s}-m_{m}}(b \sqrt{x}), \\
x & \geq 0, m_{m} \geq 0.5, m_{s}>0
\end{aligned}
$$

where $K_{m_{s}-m_{m}}($.$) is the modified Bessel function of the second$ kind and order $\left(m_{s}-m_{m}\right)$ and $b=2 \sqrt{\frac{m_{m} m_{s}}{\Omega_{0}}}$. The PDF in

appeared first in [8] to model target and clutter scattering in radar and is named later as the generalized- $K^{1}$ model or the McDaniel model in sonar literature, respectively ([5] and references therein). The cumulative distribution function (CDF) of the generalized- $K \mathrm{RV}$ can be obtained using (4) as [7]

$$
\begin{aligned}
& P(\gamma)=\frac{2}{\Gamma\left(m_{m}\right) \Gamma\left(m_{s}\right)} \pi \csc \left(\pi\left(m_{s}-m_{m}\right)\right) \\
& \times\left[\begin{array}{l}
\left(\frac{b}{2}\right)^{m_{m}} \Gamma\left(m_{m}\right) \gamma^{m}{ }_{1} F_{2}\left(m_{m} ; 1-m_{s}+m_{m} ; m_{m}+1 ; b^{2} \gamma\right)- \\
\left(\frac{b}{2}\right)^{m_{s}} \Gamma\left(m_{s}\right) \gamma^{m_{s}}{ }_{1} F_{2}\left(m_{s} ; 1+m_{s}-m_{m} ; m_{s}+1 ; b^{2} \gamma\right)
\end{array}\right],
\end{aligned}
$$

where ${ }_{p} F_{q}(\cdot, \cdot, \cdot, \cdot)$ is the generalized hyper-geometric function [10].

The moment generating function of the PDF in (4) can be derived as [7]

$$
\Phi(s)=\left(\frac{b^{2}}{4 s}\right)^{m_{s}+m_{m}-1}{ }^{2} \exp \left(-\frac{b^{2}}{8 s}\right) W_{\frac{1-m_{s}-m_{m}}{2}, \frac{m_{s}-m_{m}}{2}}\left(\frac{b^{2}}{4 s}\right)
$$

where $W_{\mu, \lambda}(z)$ is the Whittaker function [10].

However, as pointed in [11], the computation of the CDF expression in (5) is not straightforward and requires the use of approximations and asymptotic expansions or the use of the numerical inversion of the characteristic function. Moreover, further derivations using the characteristic function approach,

\footnotetext{
${ }^{1}$ It should be highlighted here that in literature, the notion "generalized- $K$ " was used to denote another similar distribution [9].
}

such as the PDF of the sum of $N$ Generalized- $K$ RVs, are quite involved even for the independent identically distributed (i.i.d.) case due the Whittaker function term [12].

\section{THE ADJUSTED MOMENT MATCHING METHOD}

An alternative simplifying approach is to consider approximating the PDF in (4) by a more tractable PDF. We propose using the Gamma distribution due to the following reasons: (i) Gamma distribution is a Type III Pearson distribution that is widely used in fitting a statistical model for positive RVs by matching the first and second moments [13], and (ii) since the PDF in (4) is a product of two Gamma PDFs and one of the Gamma PDFs will dominate for large values of $m_{m}$ or $m_{\mathrm{s}}$ [5].

The $n$th moment of the generalized- $K$ distribution can be derived as [7]

$$
E\left[X^{n}\right]=\frac{\Gamma\left(m_{m}+n\right) \Gamma\left(m_{s}+n\right)}{\Gamma\left(m_{m}\right) \Gamma\left(m_{s}\right)}\left(\frac{\Omega_{0}}{m_{m} m_{s}}\right)^{n} .
$$

Now, using the expression in (7), the first and second moments of the generalized- $K$ distribution can be obtained as

$$
E[X]=\Omega_{0},
$$

and

$$
E\left[X^{2}\right]=\frac{\left(m_{m}+1\right)\left(m_{s}+1\right)}{m_{m} m_{s}}\left(\Omega_{0}\right)^{2} .
$$

Matching these two moments to the first and second moments of the Gamma distribution results in

$$
\theta=\left[\frac{\left(m_{m}+1\right)\left(m_{s}+1\right)}{m_{m} m_{s}}-1\right] \Omega_{0}, \quad \theta>0
$$

and

$$
k=\frac{1}{\frac{\left(m_{m}+1\right)\left(m_{s}+1\right)}{m_{m} m_{s}}-1}, \quad k>0,
$$

where $\theta$ and $k$ denote the scale and shape parameters of the approximating Gamma distribution, respectively. The value of $K_{s}=\frac{\left(m_{m}+1\right)\left(m_{s}+1\right)}{m_{m} m_{s}}$, ranges from almost unity (when $m_{m}$ and $m_{s}$ are large) to infinity (when $m_{m}$ or $m_{\mathrm{s}}$ goes to zero). For $K_{s} \rightarrow 1$, the corresponding approximating Gamma PDF, has a very small scale parameter $(\theta)$ and an infinite shape parameter $(k)$ so that both the approximated and the approximating PDFs will approach a Dirac delta function (non-faded channel) even for equal values of $m_{m}$ and $m_{s}$. For smaller values of $m_{m}$ and $m_{s}$, the CDFs corresponding to both of the PDFs (for $\Omega_{0}=1$, in here and all over this paper) are shown in Fig. 1; it is observed that the approximation improves as the values of $m_{m}$ and $m_{\mathrm{s}}$ increase.

However, this approximation results in poor fitting in the lower and upper tail regions as compared to the original CDF 
as shown in Fig.s 2-4. To overcome this inaccuracy, the generalized- $K$ PDF can be approximated region-wise by introducing the following adjustment

$$
\theta_{a d}=\left[\frac{\left(m_{m}+1\right)\left(m_{s}+1\right)}{m_{m} m_{s}}-(1+\varepsilon)\right] \Omega_{0},-\infty \leq \varepsilon \leq K_{s}-1,
$$

and

$$
k_{a d}=\frac{1}{\frac{\left(m_{m}+1\right)\left(m_{s}+1\right)}{m_{m} m_{s}}-(1+\varepsilon)}, \quad-\infty \leq \varepsilon \leq K_{s}-1,
$$

where the adjustment parameter $\varepsilon$ is chosen so that PDF (CDF) of the approximating Gamma distribution becomes as close as desired to the PDF (CDF) of the generalized- $K$ distribution in $a$ certain region of interest. Moreover, since the relevant practical range of $K_{\mathrm{s}}$ is from almost unity (for non-faded channels) to 9 (for severe multipath fading and shadowing conditions where $m_{m}=0.5$ and $\left.m_{s}=0.5\right)$, the relevant range of the adjustment parameter $\varepsilon$ becomes $-c \leq \varepsilon \leq 8$. It can easily be shown that $K_{s}-1=\frac{1}{m_{m}}+\frac{1}{m_{s}}+\frac{1}{m_{m} m_{s}}$ which corresponds to the amount of fading as derived in [5].

approximate the PDF of the sum of independent generalized- $K$ RVs since the lower and upper tails of the PDF of the sum of independent RVs are due to the convolution of the lower and upper tails of the corresponding individual PDFs, respectively. So, the PDF of the sum of $N$ i.i.d. generalized- $K$ RVs can be approximated by the PDF of the sum of the approximating $N$ i.i.d. Gamma RVs. The PDF of the sum of $N$ i.i.d. Gamma RVs is another Gamma RV that is given as:

$$
\sum_{i=1}^{N} X_{i} \sim \operatorname{Gamma}\left(N k_{a d}, \theta_{a d}\right) \text {. }
$$

The obtained PDFs, for $m_{m}=2, m_{\mathrm{s}}=4$ and $\mathcal{\varepsilon}=0.2$, are shown in Fig. 5 and the plots of the CDFs corresponding to $N=1,2,3$ and 6 for $m_{m}=2$ and $m_{\mathrm{s}}=4$ are shown in Fig. 6 . These plots show that an adjustment of $\mathcal{E}=0.2$ results an approximation that improves from $N=1$ to $N=6$ where the approximating and the approximated CDFs are almost identical for $N=6$. Clearly, larger values of $\varepsilon$ will result in a more accurate approximation for $N=1,2$, and 3. The plots in Fig.s 7 and 8 indicate that the PDF of sum of i.i.d. generalized- $K$ RVs, for $N=6$, can be approximated by the PDF of the sum of the corresponding Gamma RVs whose parameters are obtained by "un-adjusted" moment matching. However, in general, "un-adjusted" moment matching does not necessarily lead, for large $N$, to a wellapproximating Gamma PDF, over all regions of the CDF, since results for small $m_{m}$ and $m_{s}$ have shown that the CCDF of the sum of the generalized- $K$ RVs decays faster than the CCDF of the sum of the approximating Gamma RVs, as shown in Fig. 8. This is due to keeping the same value of $\theta$ as $N$ increases where it should have got smaller. For the non-identically distributed case, the existing results in literature on the distribution of the sum of independent non-identically distributed Gamma RVs can be used [14].
To limit the lower limit of $\varepsilon$, we start from the observation that for the upper tail region, the approximating Gamma distribution resulting from "un-adjusted" moment-matching has a more rapidly decaying upper tail than the approximated PDF; Hence for a better approximation, the scale parameter, $\theta$, has to be increased (negative $\varepsilon$ ) and consequently the shape parameter, $k$, has to be decreased or, vice-versa. Numerical results have shown that a practical range of $\varepsilon$, for $m_{m}=0.5$ and $m_{\mathrm{s}}=0.5$, can be limited to $-4 \leq \varepsilon \leq 5.5$. Furthermore, the range of $\varepsilon$ decreases as the values of $m_{m}$ and $m_{\mathrm{s}}$ increase. For $m_{m}=4$ and $m_{\mathrm{s}}=2$, the practical range is $-0.5 \leq \varepsilon \leq 0.35$. The CDFs corresponding to the approximated and the approximating PDFs are shown in Fig.s 2-4 for $\Omega_{0}=1$ and various values of $m_{m}$ and $m_{\mathrm{s}}$. For the upper tail, the complementary CDF (CCDF), namely the region corresponding to $P(X \geq x) \leq 0.1$, is used to obtain more illustrative results. The results show that a broader range of the adjustment parameter is needed as the parameter $K_{s}$ increases indicating that the upper and lower tails are approximated by different Gamma PDFs. In general, the value of $\varepsilon$ can be varied according to the level of accuracy needed.

\section{ON THE APPROXIMATION OF THE PDF OF THE SUM OF INDEPENDENT GENERALIZEK- $K$ RVS}

The results obtained in Section III can also be used to

Remark 1: An alternative approach is to approximate the sum of independent generalized- $K$ RVs by a single Gamma RV in a single step. Matching the first and second moments of the sum of independent generalized- $K \mathrm{RVs}$ and the approximating Gamma distribution results in

$$
\begin{gathered}
\theta_{a d}^{\prime}=\frac{\sum_{i=1}^{N} K_{s i} \Omega_{0 i}{ }^{2}+2 \sum_{i=1}^{N} \sum_{j=i+1}^{N}\left(\Omega_{0 i} \Omega_{0 j}\right)}{\sum_{i=1}^{N} \Omega_{0 i}}-\sum_{i=1}^{N}\left(1+\varepsilon_{i}\right) \Omega_{0 i}, \quad \theta_{a d}^{\prime}>0 \\
\text { and } \quad k_{a d}^{\prime}=\frac{\sum_{i=1}^{N} \Omega_{i}}{\theta_{a d}^{\prime}}, \quad k_{a d}^{\prime}>0
\end{gathered}
$$

For the i.i.d. case, (14-a) and (14-b) simplify to

$$
\begin{aligned}
& \theta_{a d}^{\prime}=\left(K_{s}-(1-\varepsilon)\right) \Omega_{0}, \theta_{a d}^{\prime}>0, \\
& k_{a d}^{\prime}=\frac{N}{K_{s}-(1+\varepsilon)}, \quad k_{a d}^{\prime}>0 .
\end{aligned}
$$

So, if "adjusted" two-moment matching is to be used here, then we get the same scale and shape parameters as in (13). Note that (14-a) and (14-b) allow approximating the sum of non-identical independent generalized- $K \mathrm{RVs}$.

Remark 2: Interestingly, it was found by plotting the corresponding CDFs, that the PDF of the sum of $N$ i.i.d. generalized- $K \mathrm{RV}$ can be closely approximated by the PDF of a generalized- $K \mathrm{RV}$ whose parameters are $m_{m s u m}=N m_{m}$ and $m_{\text {ssum }}=N m_{s}$ respectively. However, further manipulations of the generalized- $K$ PDF are numerically involved, as explained 
in section II, and such an approximation is not as beneficial as the approximation using the Gamma distribution.

\section{APPLICATIONS}

The introduced region-wise approximation of the PDF of the generalized- $K$ distribution using the familiar Gamma distribution can be utilized in performance analysis of different communication schemes over composite fading channels. Here are some examples:

\section{A. Outage Probability}

The outage probability is simply the CDF of $\gamma$ and can be computed using the CDF of the approximating Gamma distribution. In [15], an expression of the outage probability, for $N=1$, alternative to the one given in [7] was developed but it is valid only for integer values of $m_{m}>1$. The approximation introduced here applies for both integer and non-integer values of $m_{m}$ and $m_{\mathrm{s}}$.

\section{B. Outage Capacity in SIMO Systems}

The outage capacity in SIMO (single-input multiple-output) systems is the probability that the instantaneous mutual information does not exceed a target rate $R$

$$
P_{\text {out }}(R)=P\left(\log _{2}\left[1+S N R \sum_{i=1}^{N} \gamma_{i}\right] \leq R\right) .
$$

Using the result in Section IV on the sum of $N$ independent generalized- $K \mathrm{RVs}$, the outage capacity can be computed for different values of $m_{m}, m_{\mathrm{s}}$, and input signal-to-noise ratios $(S N R S)$. The expression in (16) can be expressed as

$$
P_{\text {out }}(R)=P\left(\sum_{i=1}^{N} \gamma_{i} \leq \frac{2^{R}-1}{S N R}\right)=\int_{0}^{\frac{2^{R}-1}{S N R}} p_{\sum_{i} \gamma_{i}}(x) d x .
$$

The plot of the probability of outage probability versus the target rate $R$ given in Fig. 9 shows sufficient accuracy, using $\mathcal{E}=0.2$, for practical values of the probability of outage.

\section{CONCLUSIONS}

In this paper, we propose to approximate the generalized- $K$ distribution by a simple Gamma distribution through the use of the moment matching method. This approximation is enhanced by introducing an adjustment for the parameters of the approximating Gamma PDF computed by moment matching. The obtained results show that the introduced adjustment results in Gamma PDFs that closely approximate the lower and upper tail regions of the generalized- $K$ distribution. This sufficiently accurate approximation using the tractable Gamma distribution can significantly simplify the performance analysis over composite fading channels for different measures (Bit Error Rate (BER), outage capacity, etc.). An extension of this simplifying approximation will consider the case of the sum of correlated generalized- $K$ RVs. Also, more systematic approaches to determine the values of the adjustment parameter that will minimize, in a certain region of interest, the difference between the approximated and the approximating PDFs will be further investigated.

\section{REFERENCES}

[1] G. L. Stuber, Mobile Communication, $2^{\text {nd }}$ edition. Kluwer, 2003.

[2] A. Abdi and M. Kaveh, " $K$-distribution: An appropriate substitute for Rayleigh-lognormal distribution in fading-shadowing wireless channels," Electron. Lett., vol. 34, no. 9, pp. 851-852, April 1998.

[3] - , On the utility of Gamma PDF in modeling shadow fading (slow fading)," in Proc. IEEE Veh. Technol., Conf., vol. 3, pp. 2308-2312, May 1999.

[4] J. Salo, L. Vuokko, H. M. Elsallabi, and P. Vainikainen, "An additive model as a physical basis for shadow fading," IEEE Trans. Veh. Technol., vol. 56, no. 1, pp. 13-26, Jan. 2007.

[5] P. M. Shankar, "Error rates in generalized shadowed fading channels," Wireless Personal Commun., vol. 28, no. 4, pp. 233-238, Feb. 2004.

[6] I. M. Kostiĉ, "Analytical approach to performance analysis for channel subject to shadowing and fading," IEE Proc. Commun., vol. 152, no. 6 , pp. 821-827, Dec. 2005.

[7] P. S. Bithas, N. C. Sagias, P. T. Mathiopoulos, G. K. Karagiannidis, and A. A. Rontogiannis, "On the performance analysis of digital communications over generalized- $K$ fading channels," IEEE Commun. Lett., vol. 5, no. 10, pp. 353-355, May 2006.

[8] D. J. Lewinsky, "Nonstationary probabilistic target and clutter scattering models," IEEE Trans. Antennas \& Propag, vol. AP-31, no. 3, pp. 490-498, May 1983.

[9] R. Barakat, "Weak-scatterer generalization of the $K$-density function with application to Laser scattering in atmospheric turbulence," J. Opt. Soc. Am. A., vol. 3, no. 4, pp. 401-409, April 1986.

[10] I. S. Gradshteyn and I. M. Ryzhik, Table of Integrals, Series and Products, $6^{\text {th }}$ Ed. New York. Academic Press, 2000.

[11] M. Gu and D. A. Abraham, "Using McDaniel's model to represent non-Rayleigh reverberation," IEEE Trans. Oceanic Eng., vol. 26, pp. 348-357, July 2001.

[12] P. S. Bithas, P. T. Mathiopoulos, and S. A. Kotsopoulos, "Diversity reception over generalized- $K \quad\left(K_{\mathrm{G}}\right)$ fading channels," IEEE Trans.Wireless Commun., vol. 6, no 12, pp. 4238-4243, Dec. 2007.

[13] M. D. Springer, The Algebra of Random Variables. Wiley, New York, 1979.

[14] P. G. Moschopoulos, "The distribution of the sum of independent gamma random variables," Ann. Inst. Statist. Math. (Part A), vol. 37, pp. 541-544, 1985.

[15] A. Laourine, M. Alouini, S. Affes, and A. Stéphenne, "On the capacity of generalized-K fading channels," in Proc. IEEE GLOBECOM'07, pp. 3306-3310

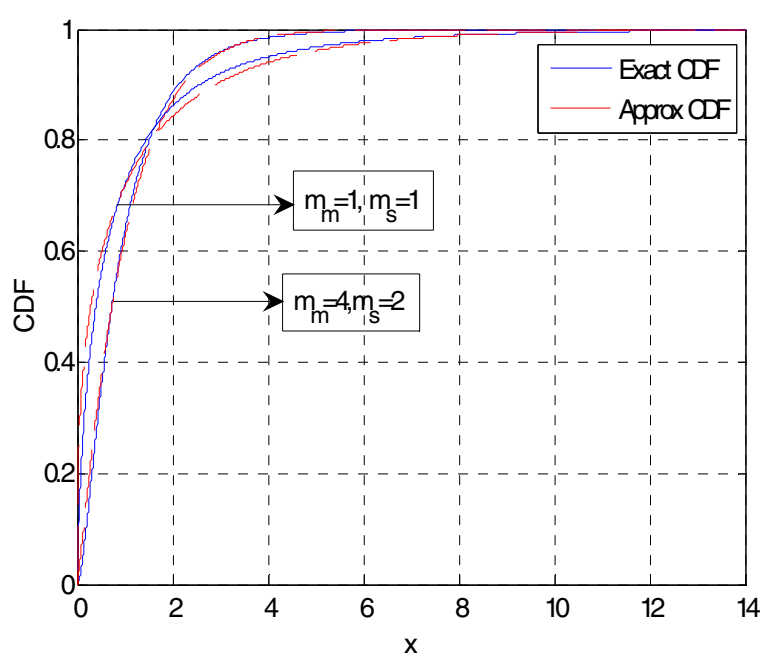

Figure 1. The plot of the CDFs corresponding to the generalized- $K$ and the approximating Gamma PDFs for different values of $m_{m}$ and $m_{\mathrm{s}}$ using "unadjusted" moment matching. 

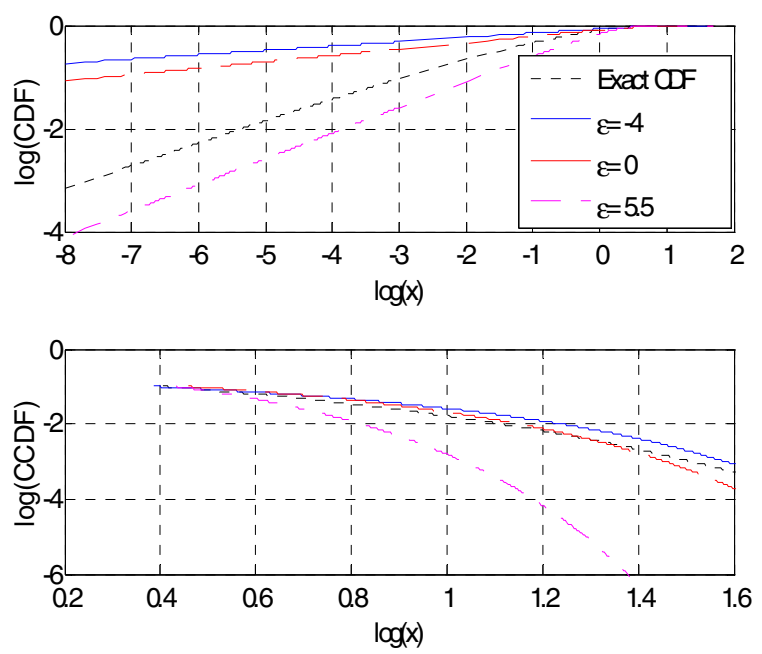

Figure 2. The plot of the lower and upper tails of the CDFs corresponding to the generalized- $K$ and the approximating Gamma PDFs for $m_{m}=0.5, m_{s}=0.5$ $\left(\sigma_{\mathrm{s}}=9.3 \mathrm{~dB}\right)$, and $\varepsilon=[-4,0,5.5]$.
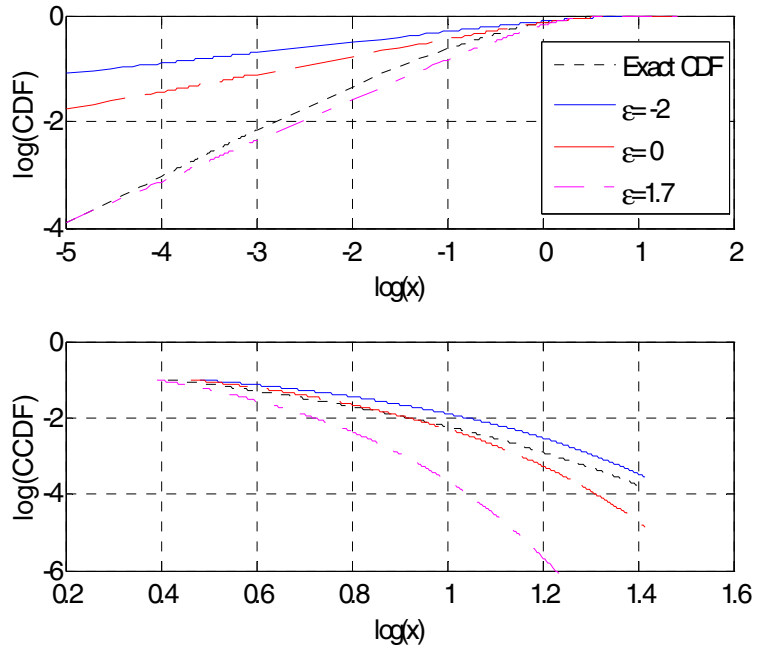

Figure 3. The plot of the lower and upper tails of the CDFs of the generalized$K$ and the approximating Gamma PDFs for $m_{m}=1, m_{\mathrm{s}}=1\left(\sigma_{\mathrm{s}}=7.4 \mathrm{~dB}\right)$ and $\varepsilon=\lceil-2,0,1.7\rceil$.
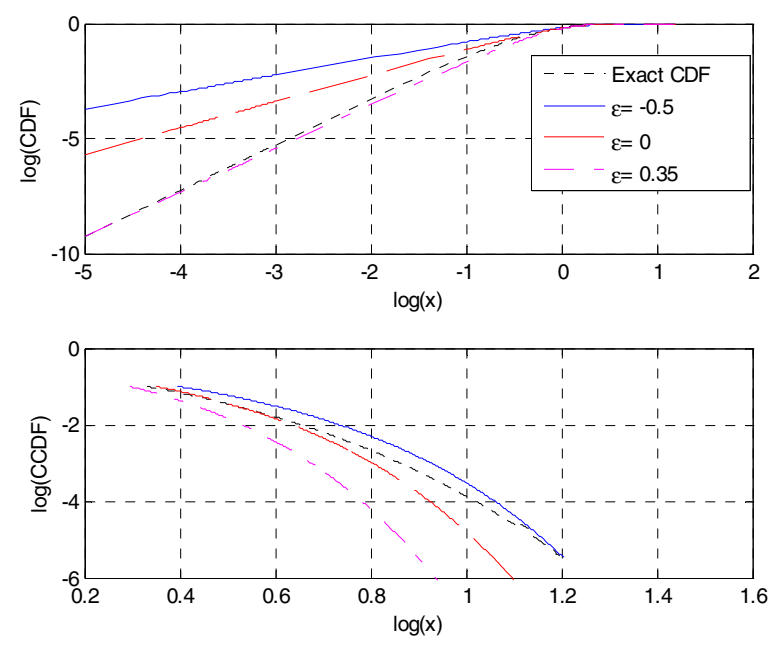

Figure 4. The plot of the lower and upper tails of the CDFs corresponding to the generalized- $K$ and the approximating Gamma PDFs for $m_{m}=4, m_{s}=2$ $(\sigma \mathrm{s}=5.6 \mathrm{~dB})$, and $\varepsilon=[-.0 .5,0,0.35]$.

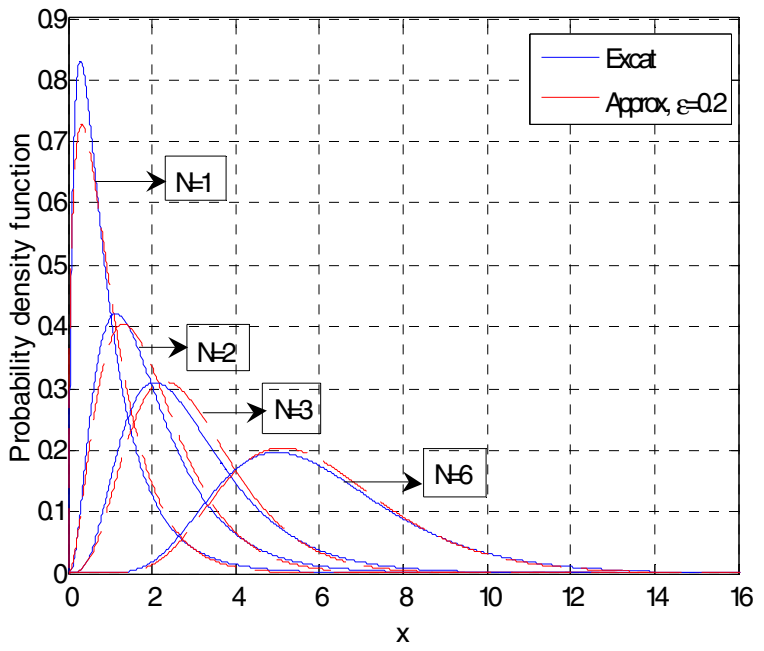

Figure 5. The plot of the PDFs of the sum of generalized- $K \mathrm{RV} s$ and the sum of the approximating Gamma RVs for $m_{m}=2, m_{\mathrm{s}}=4\left(\sigma_{\mathrm{s}}=4.2 \mathrm{~dB}\right)$ and $\varepsilon=0.2$. 


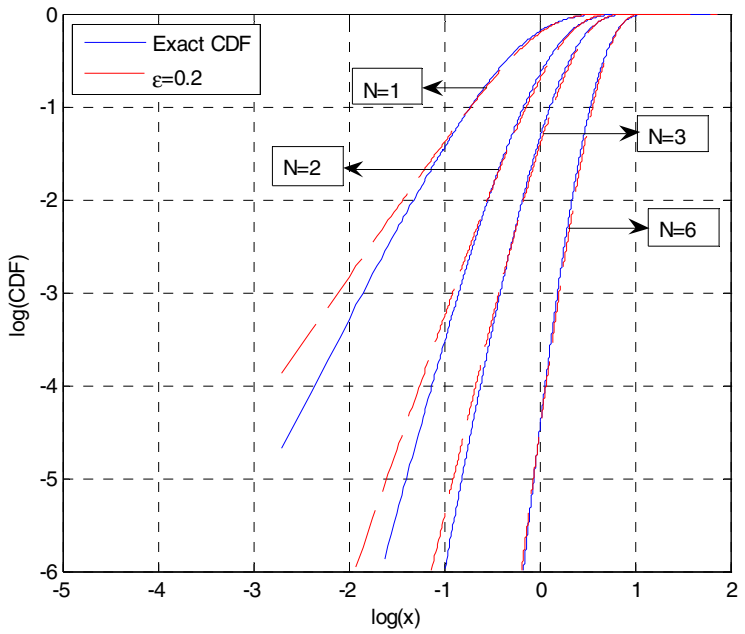

Figure 6. The plot of the lower tail of the CDFs of the sum of generalized- $K$ $\mathrm{RVs}$ and the sum of the approximating Gamma RVs for $m_{m}=2, m_{\mathrm{s}}=4\left(\sigma_{\mathrm{s}}=4.2\right.$ $\mathrm{dB})$ and $\varepsilon=0.2$.

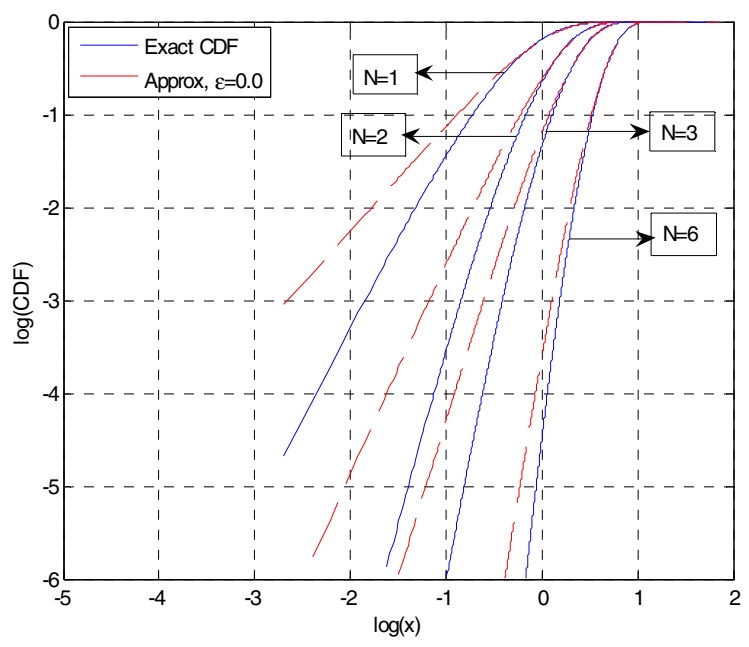

Figure 7. The plot of the lower tail of the CDFs of the sum of generalized- $K$ $\mathrm{RVs}$ and the sum of the approximating Gamma RVs for $m_{m}=2, m_{\mathrm{s}}=4, \quad\left(\sigma_{\mathrm{s}}=4.2\right.$ $\mathrm{dB}$ ) and $\varepsilon=0.0$.

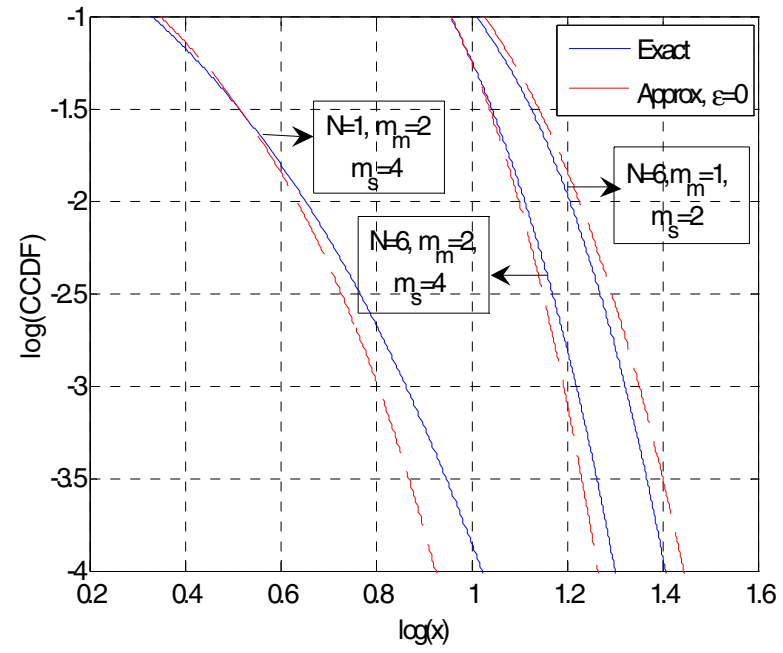

Figure 8. The plot of the upper tail of the CDFs of the sum of generalized- $K$ RVs and the sum of the approximating Gamma RVs for different values of $K_{s}$ and $\varepsilon=0.0$.

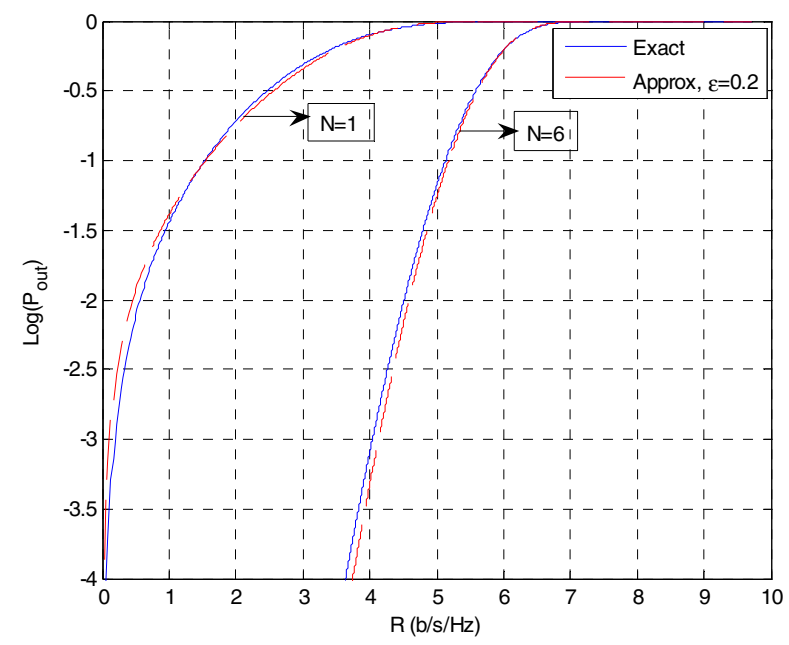

Figure 9. The plot of the $\log$ of the probability of outage for the sum of generalized- $K$ RVs and the sum of the approximating Gamma RVs for $m_{m}=2$, $m_{\mathrm{s}}=4\left(\sigma_{\mathrm{s}}=4.2 \mathrm{~dB}\right), \varepsilon=0.2$, and $\mathrm{SNR}=10 \mathrm{~dB}$. 